Supporting Information for:

\title{
Ultrafast Photodoping and Plasmon Dynamics in Fluorine-Indium Codoped Cadmium Oxide Nanocrystals for All-Optical Signal Manipulation at Optical Communication Wavelengths
}

Ilka Kriegel $^{\mathrm{a}^{*}}$, Carmine Urso ${ }^{\mathrm{a}, \mathrm{b}}$, Daniele Viola ${ }^{\mathrm{c}}$, Luca de Trizio ${ }^{\mathrm{a}}$,Francesco Scotognella ${ }^{\mathrm{a}, \mathrm{d}}$, Giulio Cerullo ${ }^{\mathrm{c}^{*}}$ and Liberato Manna ${ }^{\mathrm{a}^{*}}$

${ }^{a}$ Italian Institute of Technology, Nanochemistry Department, via Morego 30, 16163 Genova, Italy

${ }^{\mathrm{b}}$ University of Genoa, Department of Chemistry and Industrial Chemistry, via Dodecaneso 31, 16146 Genova, Italy

${ }^{\text {c }}$ Politecnico di Milano, Department of Physics, P.za Leonardo da Vinci 32, 20133 Milano, Italy

${ }^{\mathrm{d}}$ Center for Nano Science and Technology@PoliMi, Istituto Italiano di Tecnologia, Via Giovanni Pascoli 7o/3, 20133 Milano, Italy 


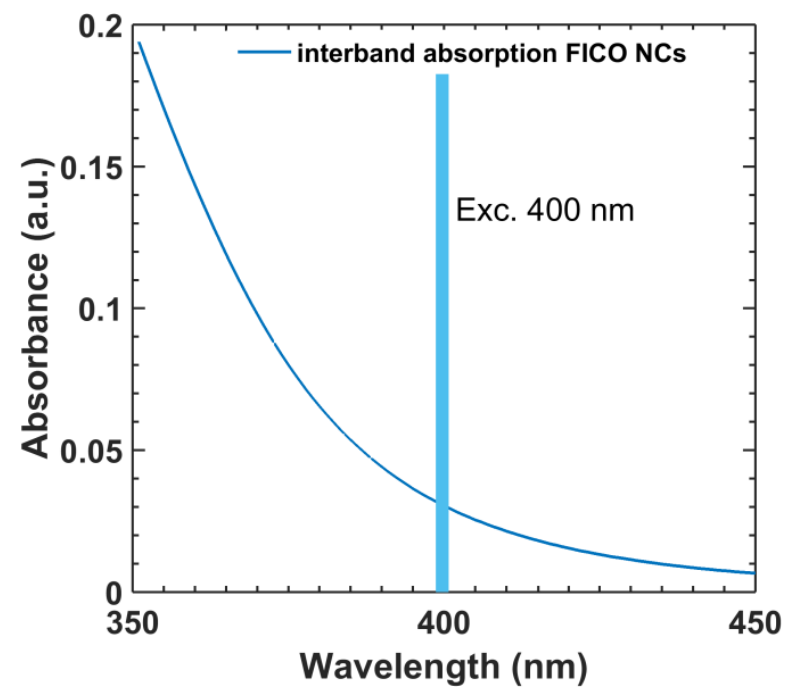

Figure S1 Interband absorption regime of the samples investigated with $400 \mathrm{~nm}$ excitation pump wavelength.

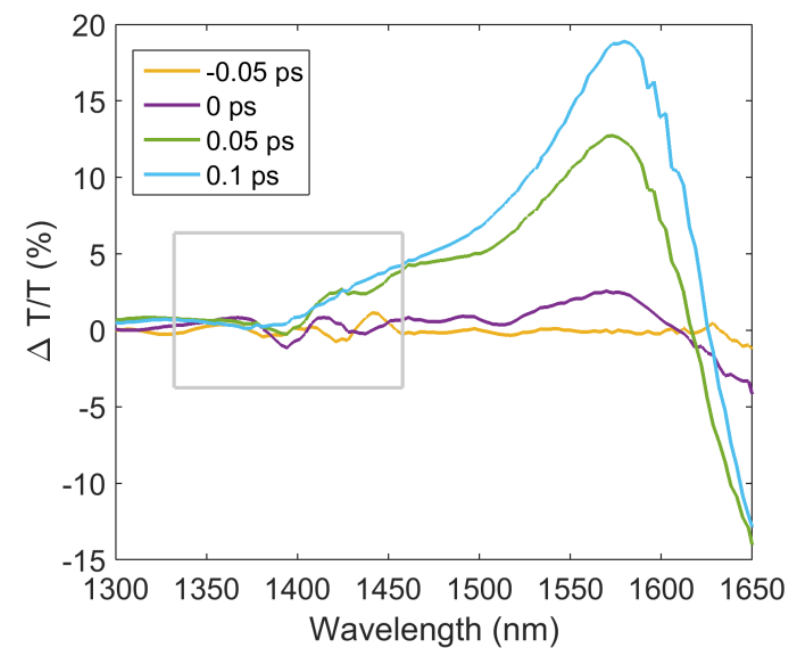

Figure S2 Various transient spectra during the rise time of the signal after intraband pumping with light pulses that have their maximum at around 1350-1400 nm showing the effect of pump scattering (see rectangular grey box). Though we do not exclude that the slight shoulder observed at 0.1 ps temporal delay is a result of an effect such as inhomogeneous broadening. 


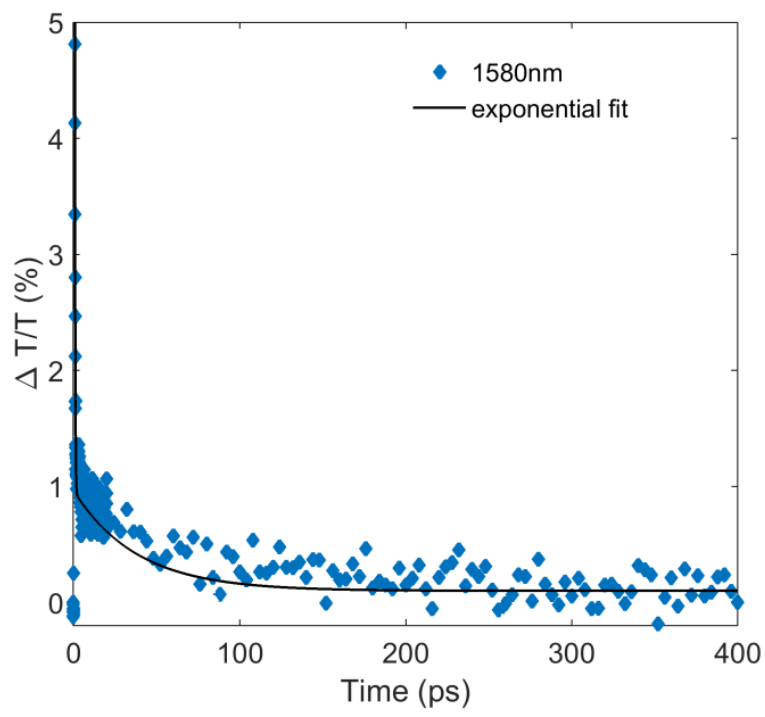

Figure S3 Transient decay dynamics at $1580 \mathrm{~nm}$ as given in the main manuscript Figure $2 \mathrm{~b}$ together with a bi-exponential fit (black and blue curve, respectively) on a longer time scale.

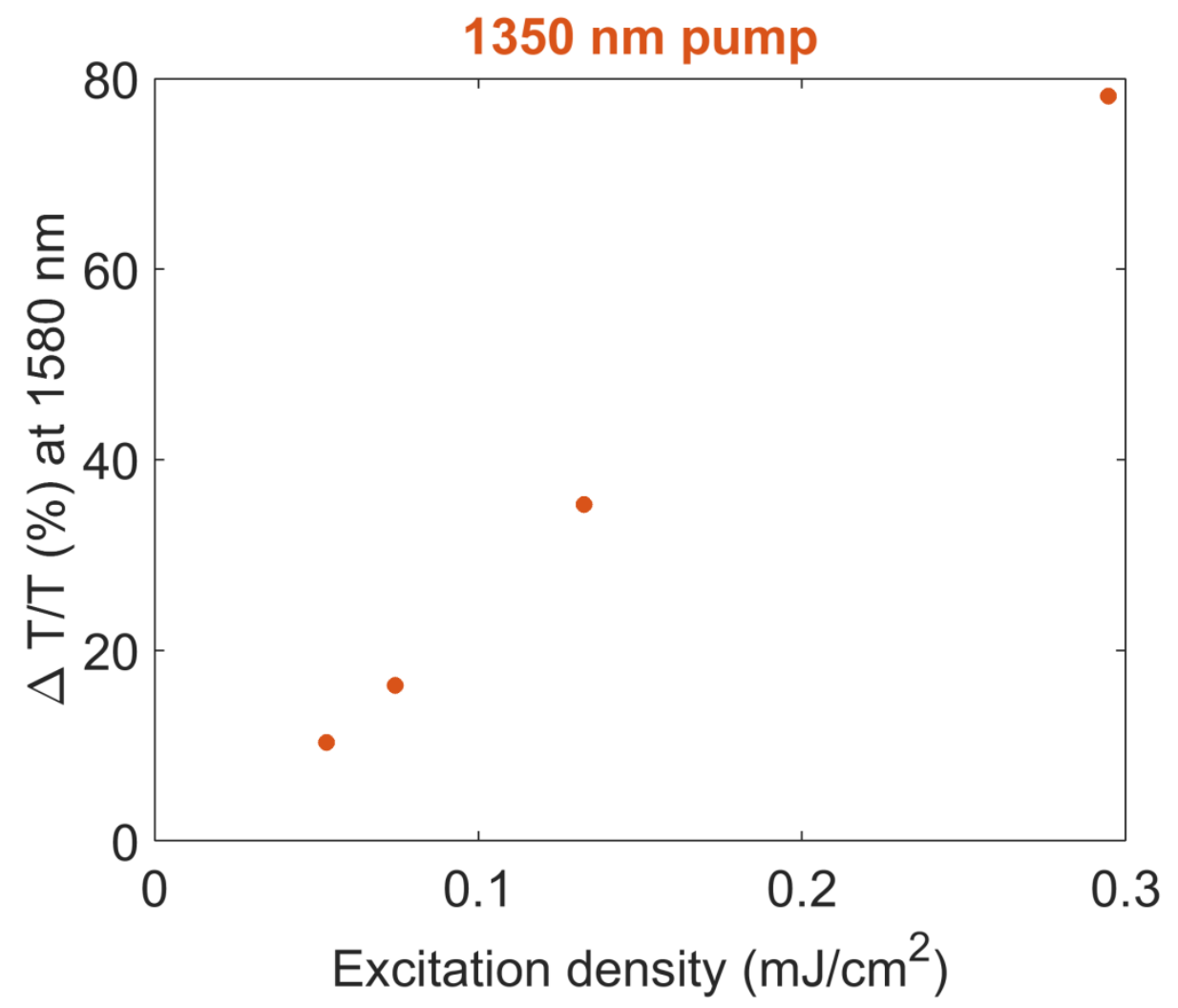

Figure $\mathbf{S}_{4}$. Plot of excitation density versus peak maximum by pumping at $1350 \mathrm{~nm}$. 


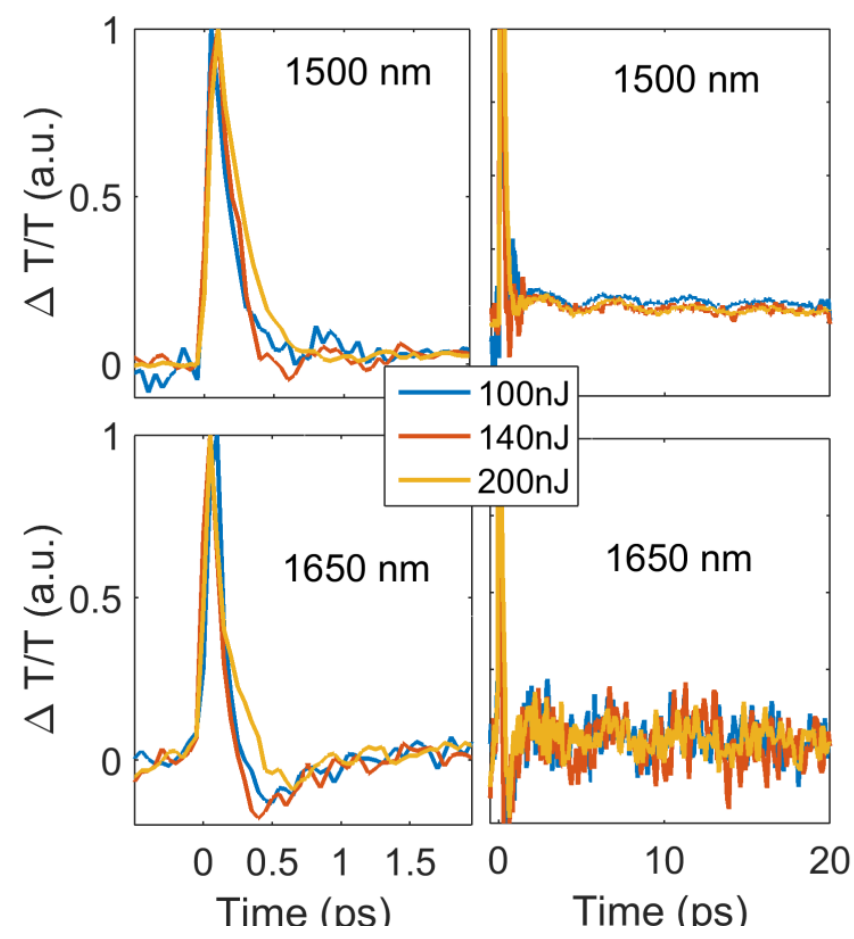

Figure $\boldsymbol{S}_{\mathbf{5}}$ Normalized decay dynamics for the pump at $1350 \mathrm{~nm}$ and probe at $1500 \mathrm{~nm}$ (upper panel) and 1650 (lower panel) for three different pump powers. The left panel is displaying the short times, showing a slight increase in the decay dynamic with increasing pump power. The right panel displays the same decay traces for longer times, showing the oscillatory behavior overlaying the two step decay.

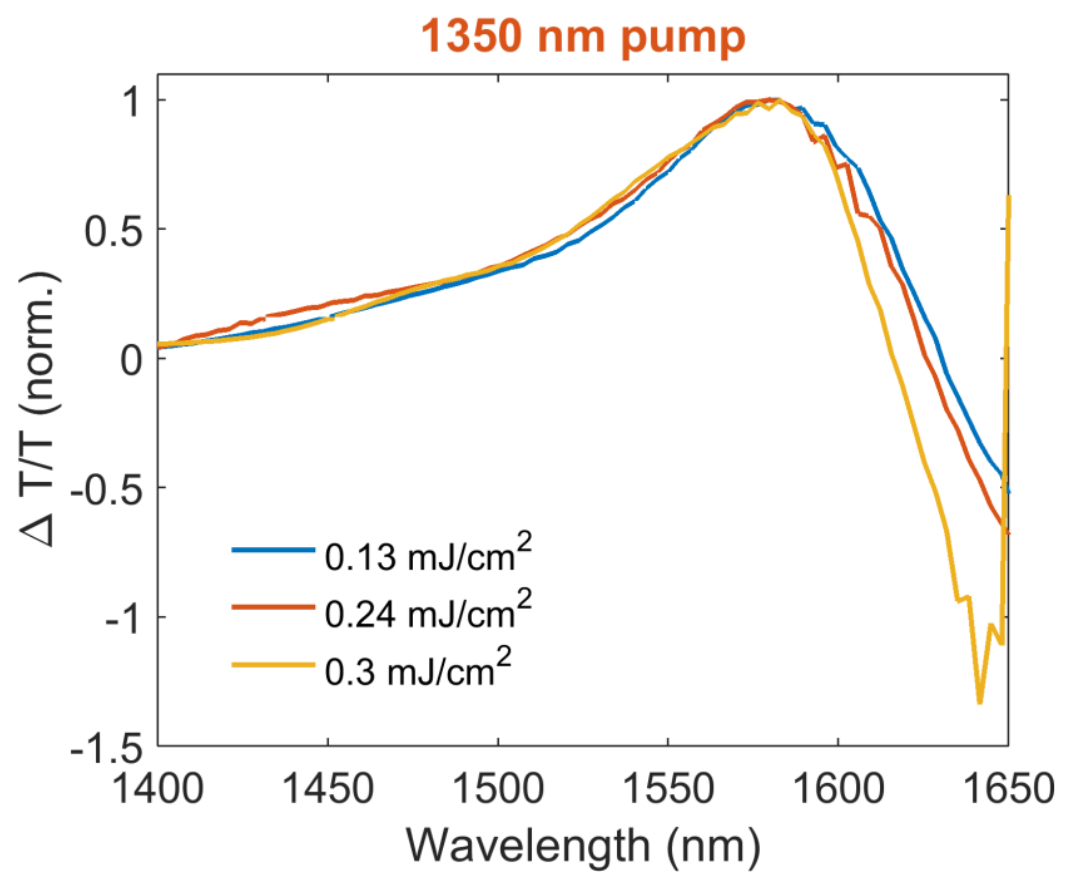

Figure S6. Spectra at $0.1 \mathrm{ps}$ after photoexcitation normalized to its maximum photobleach for three different excitation densities. It is obvious a blue shift of the spectral response with pump power. 

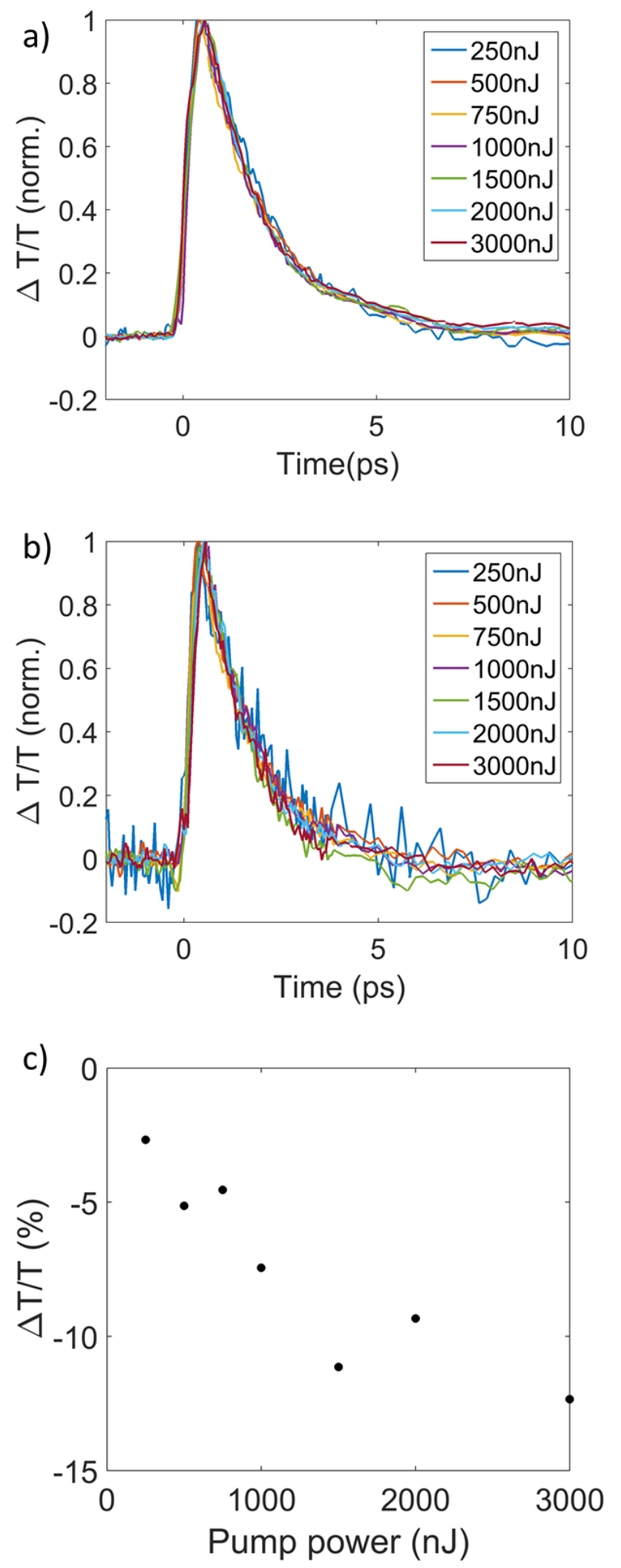

Figure S $_{7}$. Normalized decay dynamics of FICO NCs after pumping with $400 \mathrm{~nm}$ for the a) photoinduced absorption minimum and the b) bleach maximum for varying pump powers showing the fluence independence of the decay. c) $\Delta \mathrm{T} / \mathrm{T}$ intensity at minimum of the photoinduced absorption showing a nearly linear increase of the signal with pump power. The signal seems to decay versus the highest powers, which might be due to sample bleaching at high fluences. 


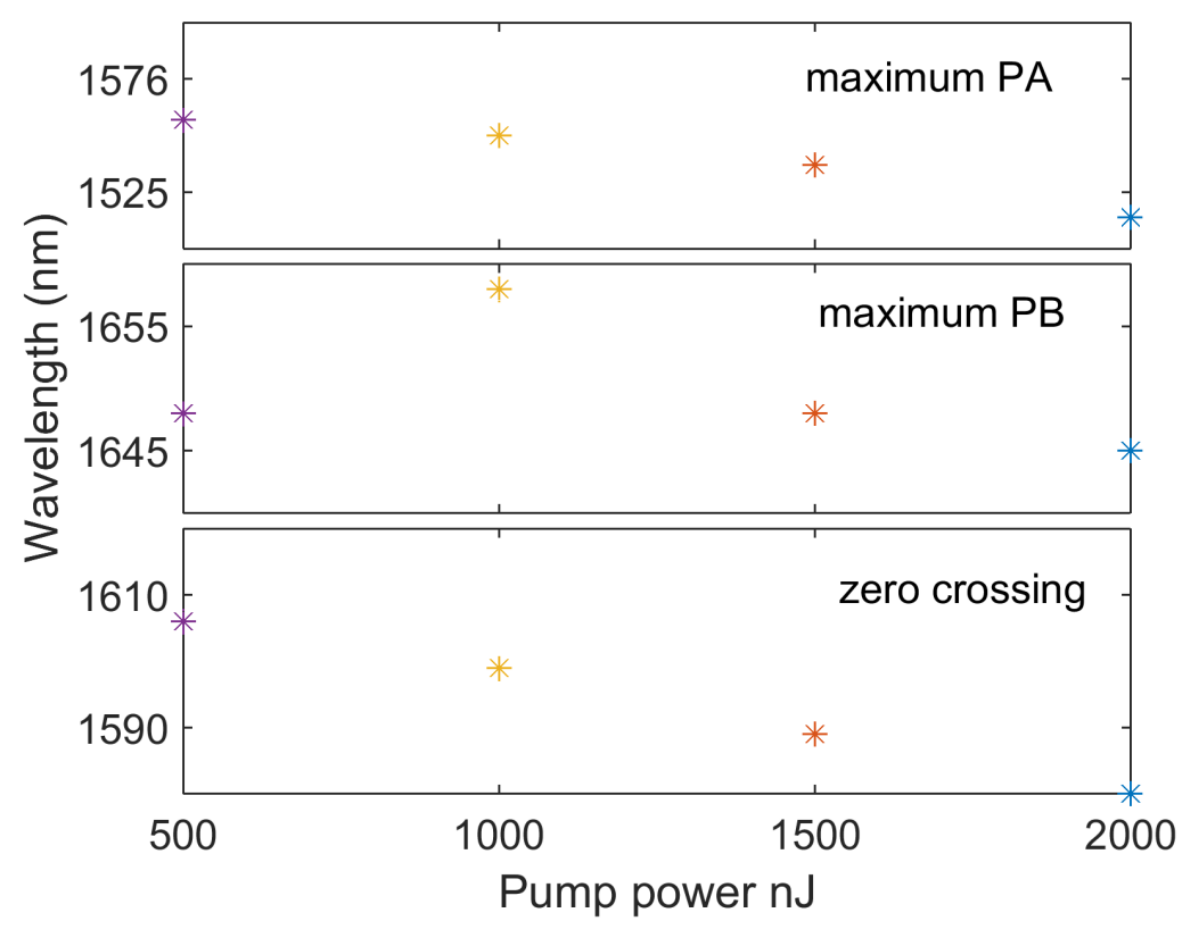

Figure S8 Summary of the peak positions of the derivative shaped TA spectra with pump power illustrating the blue-shift with increasing pump power.

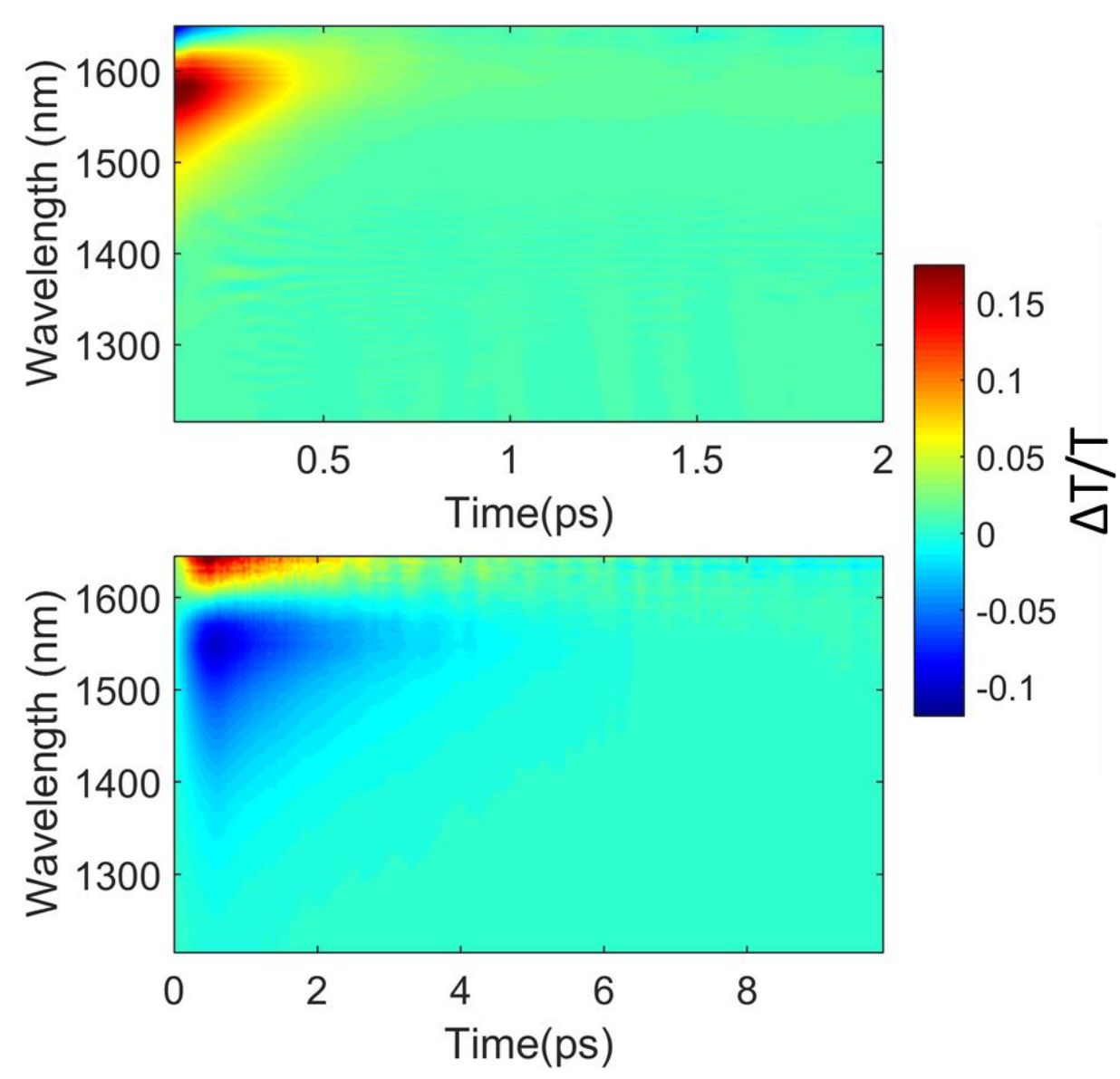

Figure S9 Experimental two-dimensional maps showing the signal as a function of pump-probe delay and probe wavelength for FICO NCs excited at $1350 \mathrm{~nm}$ (upper panel) and at $400 \mathrm{~nm}$ (lower panel) corresponding to the fit maps shown in Figure $4 \mathrm{~b}$ in the main text 

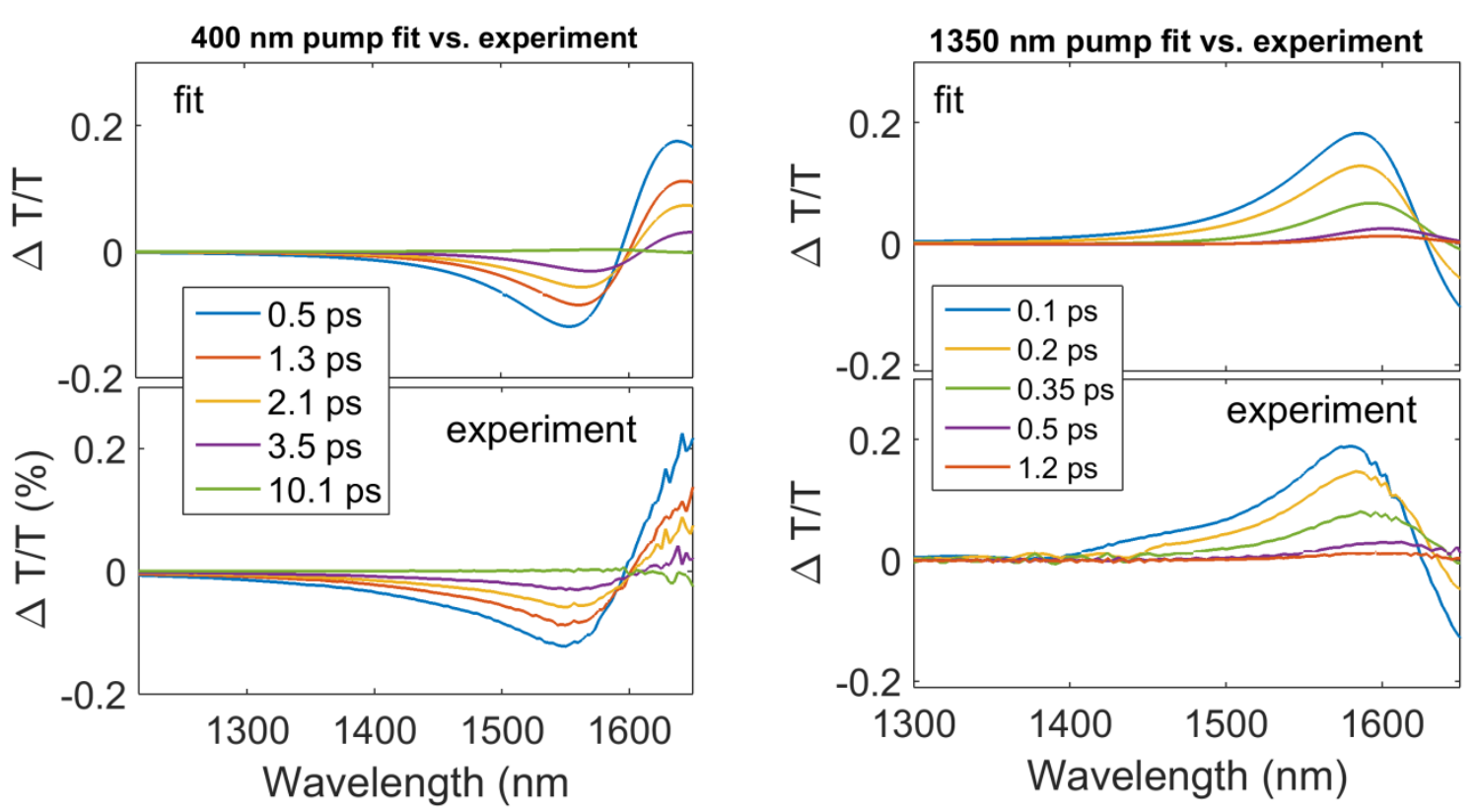

Figure S1o Transient absorption spectra (fit versus experiment) at different temporal delays for $400 \mathrm{~nm}$ pump (left) and $1350 \mathrm{~nm}$ pump (right)
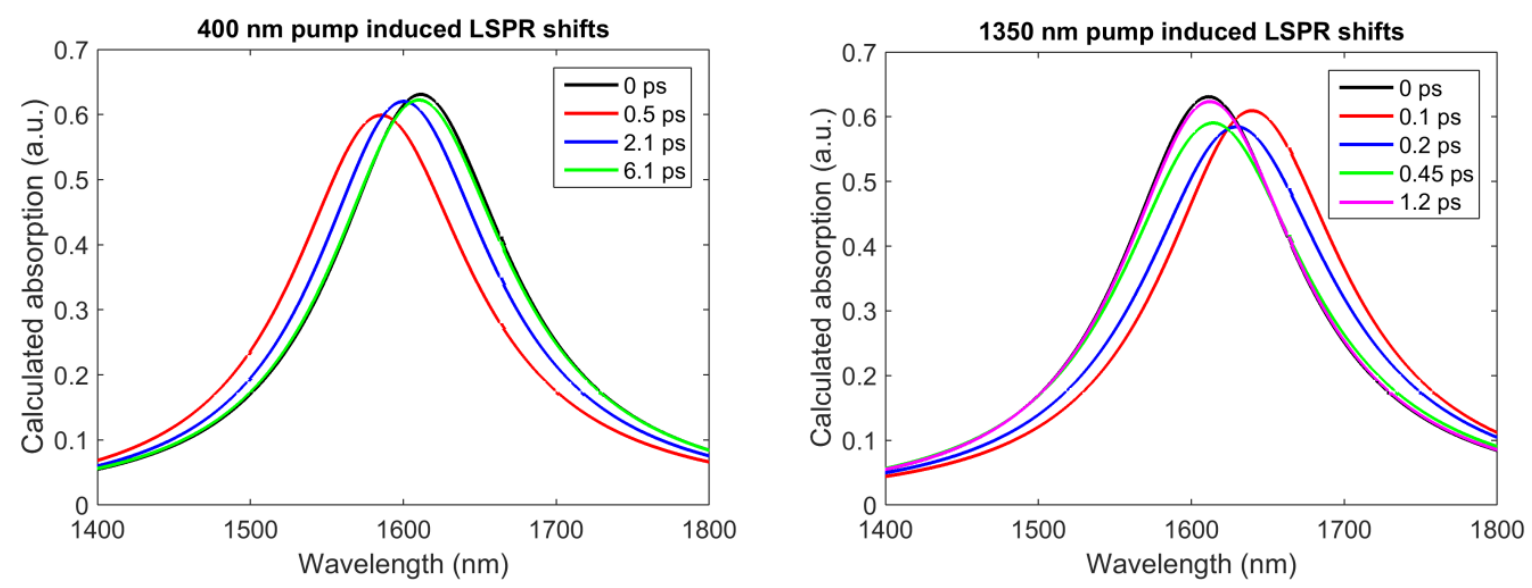

Figure S11 Calculated absorption spectra illustrating the pump induced shifts of the LSPR upon 400 nm pumping (left) and 1350 nm pumping (right).

Complete list of authors for refs. 3, 13, 16, 19:.

(3) Ni, G. X.; Wang, L.; Goldflam, M. D.; Wagner, M.; Fei, Z.; McLeod, A. S.; Liu, M. K.; Keilmann, F.; Özyilmaz, B.; Neto, A. H. C.; Hone, J.; Fogler, M. M.; Basov, D. N. Ultrafast Optical Switching of Infrared Plasmon Polaritons in High-Mobility Graphene. Nat. Photonics 2016, 10, 244-247

(13) Scotognella, F.; Della Valle, G.; Srimath Kandada, A. R.; Dorfs, D.; Zavelani-Rossi, M.; Conforti, M.; Miszta, K.; Comin, A.; Korobchevskaya, K.; Lanzani, G.; Manna, L.; Tassone, F. Plasmon Dynamics in Colloidal $\mathrm{Cu}_{2-x} \mathrm{Se}$ Nanocrystals. Nano Lett. 2011, 11, 4711-4717.

(16) Xie, Y.; Carbone, L.; Nobile, C.; Grillo, V.; D’Agostino, S.; Della Sala, F.; Giannini, C.; Altamura, D.; Oelsner, C.; Kryschi, C.; Cozzoli, P. D. Metallic-like Stoichiometric Copper Sulfide Nanocrystals: Phase- and Shape-Selective Synthesis, Near-Infrared Surface Plasmon Resonance Properties, and Their Modeling. ACS Nano 2013, 7, 7352-7369.

(19) De Trizio, L.; Gaspari, R.; Bertoni, G.; Kriegel, I.; Moretti, L.; Scotognella, F.; Maserati, L.; Zhang, Y.; Messina, G. C.; Prato, M.; Marras, S.; Cavalli, A.; Manna, L. Cuz-xP Nanocrystals as a Material Platform for Near-Infrared Plasmonics and Cation Exchange Reactions. Chem. Mater. 2015, 27, 1120-1128. 